\title{
Precision nanomedicines for prostate cancer
}

\author{
Anna Cifuentes-Rius ${ }^{1,2}$, Lisa M Butler ${ }^{3,4}$ \& Nicolas H Voelcker*,1,2,4 \\ ${ }^{1}$ Drug Delivery, Disposition \& Dynamics, Monash Institute of Pharmaceutical Sciences, Monash University, Parkville Campus, 381 \\ Royal Parade, Parkville VIC 3052, Australia \\ ${ }^{2}$ Melbourne Centre for Nanofabrication, Victorian Node of the Australian National Fabrication Facility, 151 Wellington Road, \\ Clayton, VIC 3168, Australia \\ ${ }^{3}$ Adelaide Medical School \& Freemasons Foundation Centre for Men's Health, University of Adelaide, Adelaide SA 5005, Australia \\ ${ }^{4}$ South Australian Health \& Medical Research Institute, Adelaide SA 5001, Australia \\ *Author for correspondence: nicolas.voelcker@monash.edu
}

\begin{abstract}
"Our expectation is that gaining a deeper understanding of tumor biology, together with the bio-nano interactions and biomarker availability, could allow us the development of individualized and more precise nanomedicines."
\end{abstract}

First draft submitted: 30 January 2018; Accepted for publication: 2 February 2018; Published online: 27 February 2018

Keywords: biomarkers • nanomedicine • nanotechnology • precision medicine $\bullet$ prostate cancer

\section{The importance of biomarkers for precision nanomedicine}

Over the past decade, advances in nanotechnology have enabled the production of nanomaterial-based therapeutics [1]. These nanomaterials, with a submicrometer size and high surface area, when used to diagnose and treat disease are known as nanomedicines. Nanomedicine is an interdisciplinary field combining nanotechnology and pharmaceutical and biomedical science, which offers new possibilities for the design of targeted-delivery platforms for both diagnosis and therapy [1,2]. In cancer research, this technology has the potential to revolutionize the way we manage the malignancy not only by how treatment is directed but also from a diagnosis and prognosis perspective. Such nanomedicines will allow focus on early detection of disease, combining therapies by loading multiple therapeutics into nanoscale-delivery systems, and improved tumor specificity by selective targeting and delivery.

These advances have been possible due to the parallel efforts of tumor biologists in deciphering relevant biomarker profiles of patients and/or tumors, with the ultimate goal of better personalizing nanomedicines. The NIH defines 'biomarker' as a biomolecule used as an indicator of a normal or pathologic process, or of a condition or response to a therapeutic intervention [3]. Biomarkers may be found in blood, urine and/or tissues and can comprise specific cells, molecules, proteins, genes, enzymes, hormones, or antibodies, among others. Biomarkers are classified based on their function and their biological properties, being used to identify disease, predict/monitor its state and guide targeted treatment. In nanomedicine, four types of biomarkers are mainly employed: diagnostic, prognostic, predictive and monitoring/pharmacodynamics [4]. Diagnostic biomarkers are designed to detect the presence of cancer, while prognostic biomarkers indicate certain features of the cancer and the likelihood of the patient's outcome, with or without treatment. Predictive biomarkers, on the other hand, are used to assess the likely risk or benefit of a specific clinical intervention or therapy and, therefore, improve decision making on an individual basis. Finally, pharmacodynamic biomarkers provide information about and allow monitoring of clinical responses to an intervention. It is now evident that efforts in the preclinical development of new therapies must be accompanied by biomarkers discovery and development. Some of these biomarkers are also therapeutic targets, having the potential to simultaneously influence tumor growth [5].

There are several nanoparticle formulations being currently investigated. Examples are liposomes [1], polymer nanostructures [6,7], protein constructs [1], gold nanomaterials [8,9], carbon nanotubes [10] and porous silicon nanoparticles [11-13], among others. From these nanoparticle-based formulations, few have progressed into clinical trials and/or have been approved by the US FDA [1]. While nanoparticle investigations mainly focus on the optimization of physicochemical properties of the nanoparticle constructs such as size, stiffness, surface chemistry, 
geometry, etc., the microenvironment and intrinsic heterogeneity of tumors is often overlooked. As an example, most of the FDA-approved nanotherapeutics that are administered intravenously rely on passive accumulation through the leaky vasculature present in tumors, known as the enhanced permeability and retention (EPR) effect. It is now known that the EPR effect can substantially vary between tumors types, patients and even over time, leading to difference in nanoparticle biodistribution and lack of uniformity [1]. Our expectation is that gaining a deeper understanding of tumor biology, together with the bio-nano interactions and biomarker availability, could allow us the development of individualized and more precise nanomedicines. Therefore, in precision nanomedicine, a particular tissue, cell, metabolic microenvironment or marker (i.e., cell receptor) will be the main target, using the information provided by the biomarker to generate an active-responsive diagnostic or therapeutic effect with improved precision.

With this in mind, nanoparticles can be coated with targeting moieties such as antibodies, aptamers, peptides and small molecules that bind and recognize molecules associated with cancer [2,14]. For diagnostics and imaging applications, nanoparticles can be fluorescent or contain moieties that enhance current clinically imaging techniques such as computed tomography, MRI and positron emission tomography [2]. On the other hand, improved therapeutic outcomes have been achieved using targeted nanoparticulate systems loaded with chemotherapeutics or genes, leading to more precise delivery of cargo into the target tissue [14]. Out of increasing interest is the use of multifunctional nanoparticles that allow both diagnostics and therapy, known as theranostics, providing a more precise in vivo tracking of therapeutics via bioimaging modalities.

\section{How can we more fully exploit precision nanomedicine to improve outcomes from prostate cancer treatment?}

Clinicians have employed prostate cancer biomarkers since 1930s in the management of the disease [15,16]. The discovery of PSA, a protein biomarker present in serum, revolutionized diagnosis and management of prostate cancer [15]. However, in recent years it has become apparent that PSA as a diagnostic test is not cancer-specific and lacks prognostic ability - in other words, it cannot adequately distinguish between aggressive and indolent cancers [15-17]. This issue has contributed to the considerable over-treatment of men with low-risk and indolent prostate cancer. As a result, identifying novel prostate cancer biomarkers to improve prognosis, diagnosis and monitoring of therapeutic response have become paramount to optimize drug development and patient care. Among them, of particular relevance to precision nanomedicine are cell surface protein markers, circulating tumor cells (CTCs) and genomic biomarkers.

A cell surface receptor that has dominated the literature in the past few years is PSMA $[2,14]$. PSMA is a transmembrane protein overexpressed in highly aggressive prostate cancer cells, such as LNCaP. This receptor has attracted attention given its upregulation with increased invasiveness of disease [18]. Another interesting feature, which can be exploited for precision nanomedicine, is that it has a signal that enables the protein on the cell surface to be internalized into an endosomal compartment [19]. Altogether, this has made PSMA a powerful antigenic target used for diagnosis, imaging, prediction and therapeutic interventions of prostate cancer. Different anti-PSMA antibodies, aptamers, peptides and small molecules (e.g., glutamate urea or folic acid) have been investigated as active targeting moieties for nanomedicines $[2,6,14]$. These targeting moieties can be grafted onto a nanoparticle surface, allowing selective recognition and binding to prostate cancer cells and tissues. This approach has enabled the development of several nanoparticle probes able to detect and/or treat prostate cancer murine models $[2,6,20]$.

Despite the promising advancements in drug delivery and imaging nanomedicines for prostate cancer using PSMA as a target, the consequence of the intrinsic heterogeneity of the disease is that not all prostate cancer cells overexpress this receptor and PSMA expression varies with the aggressiveness and stage of the malignancy [18]. This difference highlights the main pitfall of a strategy that exclusively relies on PSMA targeting, and we propose that further target biomarkers should be considered to maximize the efficacy of personalized nanomedicines for a broader cross-section of patients. To this end, high levels of tyrosine kinase activity have been found in several tumor cells, including those from prostate cancer [21]. From this family, EphA2 transmembrane receptor, present in the epithelial and endothelial cells, has shown upregulation on aggressive carcinomas, and is emerging as an interesting target for nanomedicine delivery that could also reduce tumor growth and metastasis [7,21]. Having said that, however, we have only found one example using an anti-EphA2 nanotheranostics for prostate cancer, which interestingly shows higher selectivity toward the receptor target when compared with the same nanomaterial targeted to PSMA [7]. 
Another targeting strategy that has been investigated in cancer therapy is angiogenesis, since solid tumors growth depends on the formation of new blood vessels [14]. Prostate cancer is no exception, and angiogenesis is one of the key processes involved in tumor growth and metastasis [22]. One of the most proangiogenic factors is VEGF, which is commonly overexpressed in prostate cancer samples compared with benign tissue and is present in metastatic disease or castration-resistant prostate cancer [22]. However, this targeting strategy has not been fully exploited in precision nanomedicine for prostate cancer. VEGF could be targeted not only by conjugating a monoclonal antibody to the nanomedicine, but also by using the nanomedicine to deliver a siRNA with an antiangiogenic gene effect to suppress tumor growth [23].

In many cases, hypoxia and angiogenesis go hand in hand as poor oxygenation is a strong driver of tumor neovascularization [24]. Hypoxia can lead to cancer metastasis and treatment failure due to chemo- and radioresistance $[24,25]$. Because of the difficulty of drug delivery to hypoxic tumor cells that are far from the vasculature, targeting hypoxia has recently emerged as a promising tool in precision nanomedicine. This may reflect the ability of some nanoparticles to penetrate deeper within the tumor, combined with the conjugation of tumorpenetrating peptides [26], while 'smart' nanomaterials have also been developed to release cargo in response to hypoxic signals $[27,28]$. The most commonly exploited hypoxia-related biomarker is HIF-1, which is activated under hypoxic conditions [25]. Nanomedicine offers the possibility to target HIF-1 by coupling HIF-1 inhibitors such as siRNA to the nanoparticles [28,29]. These encouraging results, however, have only been seen for other cancer types like glioblastoma, and the strategy has yet to be applied to prostate cancer.

Imaging and therapeutic targeting of metastatic cells and micro-metastatic deposits is paramount to improving prognostication, staging and treatment of advanced or high-risk prostate cancer [30]. With the increasing clinical focus on detection and molecular profiling of CTCs, detecting and targeting CTCs using nanomedicine is a promising approach $[2,31]$. EpCAM is a cell surface antigen expressed in CTCs and the main target biomarker found in the literature [31,32]. Recently, the conjugation of anti-EpCAM antibodies to nanomedicines has been exploited to target CTCs with interesting results in, for example, breast cancer [32]. The emerging data on genomic and transcriptomic profiling of these rare circulating cells, which are often distinct from the primary tumor, will underpin the design and implementation of more sophisticated and individualized nanomedicine strategies to improve patient outcomes.

\section{Conclusion}

The use of precision nanomedicine in prostate cancer has great potential as a novel approach to combat the limitations of current therapies in both localized and metastatic disease settings. However, in order to achieve this potential, we need to broaden our horizons and start exploring new ways to target these nanomedicines with improved precision. Thus far, researchers have been mainly relying in either passive accumulation via EPR effect or active targeting through the recognition of PSMA receptors. However, the discovery and development of other targeting ligands and responsive nanoconjugates, combined or alone, will be necessary to address the marked heterogeneity that is inherent to prostate tumors and between patients. We also argue that the expansion of these research efforts must include use of preclinical models that incorporate the heterogeneity and complexity of clinical prostate tumors, rather than immortalized cell lines that lack relevance to clinical disease. This will necessitate collaboration between nanobiomaterialists and tumors biologists to ensure the development of effective nanomedicines with optimal properties and identify and validate more clinically-relevant biomarkers that can be translated to personalized tumor targeting and treatment.

\section{Financial \& competing interests disclosure}

This work was supported by the Prostate Cancer Foundation of Australia (NCG 1816 to LM Butler, A Cifuentes-Rius, and NH Voelcker) and the National Health and Medical Research Council (NHMRC) of Australia (GNT1112432 to A Cifuentes-Rius). LM Butler is supported by a Future Fellowship from the Australian Research Council (FT130101004). The authors have no other relevant affiliations or financial involvement with any organization or entity with a financial interest in or financial conflict with the subject matter or materials discussed in the manuscript apart from those disclosed.

No writing assistance was utilized in the production of this manuscript. 


\section{References}

1. Shi J, Kantoff PW, Wooster R, Farokhzad OC. Cancer nanomedicine: progress: challenges and opportunities. Nat. Rev. Cancer 17, 20 (2016).

2. Chinen AB, Guan CM, Ferrer JR, Barnaby SN, Merkel TJ, Mirkin CA. Nanoparticle probes for the detection of cancer biomarkers: cells: and tissues by fluorescence. Chem. Rev. 115(19), 10530-10574 (2015).

3. Howat WJ, Lewis A, Jones P et al. Antibody validation of immunohistochemistry for biomarker discovery: recommendations of a consortium of academic and pharmaceutical based histopathology researchers. Methods 70(1), 34-38 (2014).

4. Jia S. Prostate cancer - a biomarker perspective. Front. Endocrinol. (Lausanne) 3, 72 (2012).

5. Shen Z. Cancer biomarkers and targeted therapies. Cell Biosci. 3, 6 (2013).

6. Fuchs AV, Tse BWC, Pearce AK et al. Evaluation of polymeric nanomedicines targeted to PSMA: effect of ligand on targeting efficiency. Biomacromolecules 16(10), 3235-3247 (2015).

7. Pearce AK, Fuchs AV, Fletcher NL, Thurecht KJ. Targeting nanomedicines to prostate cancer: evaluation of specificity of ligands to two different receptors in vivo. Pharm. Res. 33(10), 2388-2399 (2016).

8. Cifuentes-Rius A, De Puig H, Kah JCY, Borros S, Hamad-Schifferli K. Optimizing the properties of the protein corona surrounding nanoparticles for tuning payload release. ACS Nano 7(11), 10066-10074 (2013).

9. Cifuentes-Rius A, Ivask A, Das S et al. Gold nanocluster-mediated cellular death under electromagnetic radiation. ACS Appl. Mater. Interfaces 9(47), 41159-41167 (2017).

10. Cifuentes-Rius A, Boase NRB, Font I et al. In vivo fate of carbon nanotubes with different physicochemical properties for gene delivery applications. ACS Appl. Mater. Interfaces 9(13), 11461-11471 (2017).

11. Alhmoud H, Cifuentes-Rius A, Delalat B, Lancaster DG, Voelcker NH. Gold-decorated porous silicon nanopillars for targeted hyperthermal treatment of bacterial infections. ACS Appl. Mater. Interfaces 9(39), 33707-33716 (2017).

12. Cifuentes-Rius A, Ivask A, Sporleder E et al. Dual-action cancer therapy with targeted porous silicon nanovectors. Small 13(29), 1701201 (2017).

13. Sinha S, Tong WY, Williamson NH et al. Novel GD-loaded silicon nanohybrid: a potential epidermal growth factor receptor expressing cancer cell targeting magnetic resonance imaging contrast agent. ACS Appl. Mater. Interfaces 9(49), 42601-42611 (2017).

14. Byrne JD, Betancourt T, Brannon-Peppas L. Active targeting schemes for nanoparticle systems in cancer therapeutics. Adv. Drug Deliv. Rev. 60(15), 1615-1626 (2008).

15. Prensner JR, Rubin MA, Wei JT, Chinnaiyan AM. Beyond PSA, the next generation of prostate cancer biomarkers. Sci. Transl. Med. 4(127), 127rv123-127rv123 (2012).

16. Gaudreau P-O, Stagg J, Soulières D, Saad F. The present and future of biomarkers in prostate cancer: proteomics: genomics: and immunology advancements. Biomark. Cancer 8(Suppl. 2), 15-33 (2016).

17. Terada N, Akamatsu S, Kobayashi T, Inoue T, Ogawa O, Antonarakis ES. Prognostic and predictive biomarkers in prostate cancer: latest evidence and clinical implications. Ther. Adv. Med. Oncol. 9(8), 565-573 (2017).

18. Wright GL Jr, Haley C, Beckett ML, Schellhammer PF. Expression of prostate-specific membrane antigen in normal: benign: and malignant prostate tissues. Urol. Oncol. Semin. Ori. 1(1), 18-28 (1995).

19. Chang SS. Overview of prostate-specific membrane antigen. Rev. Urol. 6(Suppl. 10), S13-S18 (2004).

20. Kim D, Jeong YY, Jon S. A drug-loaded aptamer-gold nanoparticle bioconjugate for combined CT imaging and therapy of prostate cancer. ACS Nano 4(7), 3689-3696 (2010).

21. Chen P, Huang YAN, Zhang BO, Wang Q, Bai P. EphA2 enhances the proliferation and invasion ability of LNCaP prostate cancer cells. Oncol. Lett. 8(1), 41-46 (2014).

22. Corcoran NM, Gleave ME. Targeted therapy in prostate cancer. Histopathology 60(1), 216-231 (2012).

23. Kim SH, Jeong JH, Lee SH, Kim SW, Park TG. Local and systemic delivery of VEGF siRNA using polyelectrolyte complex micelles for effective treatment of cancer. J. Control. Release 129(2), 107-116 (2008).

24. Moeller BJ, Cao Y, Vujaskovic Z, Li CY, Haroon ZA, Dewhirst MW. The relationship between hypoxia and angiogenesis. Semin. Radiat. Oncol. 14(3), 215-221 (2004).

25. Deep G, Panigrahi GK. Hypoxia-induced signaling promotes prostate cancer progression: exosomes role as messenger of hypoxic response in tumor microenvironment. Crit. Rev. Oncog. 20(5-6), 419-434 (2015).

26. Wang Y, Xie Y, Li J et al. Tumor-penetrating nanoparticles for enhanced anticancer activity of combined photodynamic and hypoxia-activated therapy. ACS Nano 11(2), 2227-2238 (2017).

27. Thambi T, Deepagan VG, Yoon HY et al. Hypoxia-responsive polymeric nanoparticles for tumor-targeted drug delivery. Biomaterials 35(5), 1735-1743 (2014).

28. Aldea M, Florian IA, Kacso G et al. Nanoparticles for targeting intratumoral hypoxia: exploiting a potential weakness of glioblastoma. Pharm. Res. 33(9), 2059-2077 (2016). 
29. Jahanban-Esfahlan R, De La Guardia M, Ahmadi D, Yousefi B. Modulating tumor hypoxia by nanomedicine for effective cancer therapy. J. Cell. Physiol. Suppl. 233(3), 2019-2031 (2018).

30. Massoner P, Thomm T, Mack B et al. EpCAM is overexpressed in local and metastatic prostate cancer: suppressed by chemotherapy and modulated by MET-associated miRNA-200c/205. Br. J. Cancer 111, 955 (2014).

31. Simon M, Stefan N, Plückthun A, Zangemeister-Wittke U. Epithelial cell adhesion molecule-targeted drug delivery for cancer therapy. Expert Opin. Drug Deliv. 10(4), 451-468 (2013).

32. Jenkins SV, Nima ZA, Vang KB et al. Triple-negative breast cancer targeting and killing by EpCAM-directed: plasmonically active nanodrug systems. NPJ Precis. Oncol. 1(1), 27 (2017). 
